\title{
Utilizando Espectroscopia de Energia Dispersiva (EDS) para Comparação de Medicamentos Genéricos e Similar com o seu Referência
}

\section{The Use of Energy Dispersive Spectroscopy (EDS) For Comparison of Generic and Similar Drugs with Their Reference Drugs}

\author{
LEANDRO DA CONCEIÇÃO LUIZ1 \\ CARLADA SILVA FREIRES VARELLA ${ }^{2}$ \\ DEBORAH DEMARQUE MARTINS DA SILVA ${ }^{2}$ \\ DIANA LOURENÇO BRANDÃO ${ }^{3}$ \\ RAFAELATAVARES BATISTA ${ }^{4}$ \\ RENATO PEREIRADE FREITAS 5
}

\section{RESUMO}

Introdução: Os medicamentos genéricos devem apresentar mesmo princípio ativo que o seu referência, na mesma concentração, dose e forma farmacêutica. Normalmente o seu valor é inferior, assim, muitas pessoas questionam: sua composição, eficiência e tempo de resposta. O Microscópio Eletrônico de Varredura (MEV) produz imagens de alta ampliação e resolução através da interação de elétrons com os átomos da amostra. Nele também é possível realizar uma análise multielementar através de um sistema de Espectroscopia por Energia Dispersiva (EDS). Objetivo: Visto a característica do MEV/EDS e o questionamento levantado pelo uso de genéricos, realizou-se uma análise qualitativa do medicamento referência, genéricos e similar do Diclofenaco de Potássio $50 \mathrm{mg}$ a fim de verificar suas composições e compará-las. Material e Métodos: Utilizou-se medicamentos genéricos de 3 indústrias farmacêuticas distintas, o similar e o seu referência. As amostras foram irradiadas durante 300s no MEV/EDS TM-3000 Hitachi, com 3 medidas em pontos distintos de cada comprimido. As medidas também foram feitas com os medicamentos sem os revestimentos. Resultados: A técnica conseguiu verificar tanto os elementos presentes nos excipientes quanto no princípio ativo do medicamento. Foi visto que alguns se distinguiam quando irradiado com o revestimento, mas ao retirar o mesmo, todos os genéricos apresentaram-se bem semelhantes qualitativamente quando comparados ao medicamento de referência. Conclusões: A técnica mostrou-se eficiente e adequada para as amostras, pois através dos espectros dos fármacos genéricos e similar foi possível identificar não somente o princípio ativo, mas também seus excipientes e compará-los ao fármaco referência.

DESCRITORES

Fármacos. Genéricos. Diclofenaco de Potássio. Espectroscopia.

\begin{abstract}
Introduction: Generic drugs should have the same active ingredient than their reference drugs, at the same concentration, dose and dosage form. As the costs of generics are lower, many people have raised questions about their composition, efficiency and response time. A Scanning Electron Microscope (SEM) produces high magnification and resolution images through the interaction of electrons with the sample atoms. This equipment may also be used to perform a multielement analysis using an Energy Dispersive Spectroscopy (EDS) system. Objective: Given the SEM/EDS features and the questions raised on the use of generics, we carried out a qualitative analysis of the reference, generic and similar drugs of Potassium Diclofenac $(50 \mathrm{mg})$, in order to verify their compositions and compare them. Materials and Methods: Three different brands of each drug (reference, generic and similar) were tested. The samples were irradiated for 300 seconds using a SEM/EDS Hitachi TM-3000 microscope, with three measurements at different points in each tablet. The measurements were also made with the drug without the coating Results: The technique was able to detect the constituents present in both the excipients and the active ingredient of the drug. Some differences were found when the samples were irradiated with their coating. On the other hand, all generic drugs were found to be qualitatively similar to their reference drugs when the coating was removed. Conclusions: The technique was proven efficient and suitable for the samples, because through the spectra of the generic and similar drugs it was possible to identify not only the active principle but also the excipients, and compare them to the reference drug.
\end{abstract}

DESCRIPTORS

Drug. Generic. Diclofenac Potassium. Spectroscopy.

1 Professor Adjunto do Curso de Farmácia da Faculdade Bezerra de Araújo (FABA), Rio de Janeiro/RJ. Doutorando em Física pelo Departamento de Física da Universidade Federal de Juiz de Fora (UFJF), Juiz de Fora/MG, Brasil.

2 Estudante do curso de Farmácia da Faculdade Bezerra de Araújo (FABA), Rio de Janeiro/RJ, Brasil.

3 Farmacêutica bioquímica. Especialista em Proteção Radiológica e Segurança de Fontes pelo Instituto de Radioproteção e Dosimetria da Comissão Nacional de Energia Nuclear (IRD/CNEN), Rio de Janeiro/RJ, Brasil.

4 Doutoranda em Física pelo Instituto de Física Armando Dias Tavares da Universidade do Estado do Rio de Janeiro (IFADT/UERJ), Rio de Janeiro Brasil. Colaboradora do Instituto Federal de Educação, Ciência e Tecnologia do Rio de Janeiro, Campus Paracambi (IFRJ), Paracambi/RJ, Brasil.

5 Doutor em Ciências pelo Programa de Engenharia Nuclear do Instituto Alberto Luiz Coimbra de Pós-Graduação e Pesquisa de Engenharia da Universidade Federal do Rio de Janeiro (COPPE/UFRJ). Rio de Janeiro/RJ, Brasil. Professor do Ensino Técnico e Tecnológico do Instituto Federal de Educação, Ciência e Tecnologia do Rio de Janeiro, Campus Paracambi (IFRJ). Paracambi/RJ, Brasil. 
$\mathrm{E}$ m 1999, os medicamentos genéricos foram introduzidos no Brasil, através da Lei 9.787 (10/ 02/99) da Agência Nacional de Vigilância Sanitária, do Ministério da Saúde (ANVISA/MS). Um medicamento genérico é aquele que deve apresentar o princípio ativo, dose e forma farmacêutica, na mesma concentração do seu referência. Deve ainda ser administrado pela mesma via e com a mesma indicação terapêutica do medicamento de referência. Ele deve apresentar intercambialidade, isto é, a segura substituição do medicamento referência pelo seu genérico. A intercambialidade é assegurada por testes de equivalência terapêutica, que incluem comparação in vidro, através dos estudos de equivalência farmacêutica e in vivo, com os estudos de bioequivalência apresentados à ANVISA. Já o similar pode não ter a mesma forma farmacêutica e excipientes, por isso não tem a mesma biodisponibilidade e bioequivalência que o medicamento de referência, já que formas farmacêuticas diferentes têm diferentes locais de absorção e de tempo de início de ação. A substituição do medicamento prescrito pelo medicamento genérico correspondente, somente pode ser realizada pelo farmacêutico responsável pela farmácia ou drogaria e deverá ser registrada na prescrição médica. Enquanto aos medicamentos similares, estes não podem ser oferecidos como substitutos dos medicamentos de referência, mesmo que eles tenham o mesmo resultado. Prescrever um medicamento similar é exclusividade do médico.

Os medicamentos genéricos são identificados por sua embalagem, que deve conter: o nome do princípio ativo, o título: "Medicamento Genérico - Lei n' 9787/99”, uma tarja amarela, com um “G”, em cor azul em destaque, conforme definição publicada na Resolução n 47, de 28 de março de 2001.

Em 2014, a Associação Brasileira das Indústrias de Medicamentos Genéricos (PróGenéricos) afirmou que o mercado de medicamentos genéricos, tinha $28,2 \%$ de participação no mercado farmacêutico, e que $85 \%$ dos produtos dispensados pelo Programa Farmácia Popular são medicamentos genéricos. Ainda de acordo com a associação, há medicamentos genéricos disponíveis para mais de $95 \%$ das doenças conhecidas, seja ela simples ou complexa. Os anti-hipertensivos corresponderam $60 \%$ de participação em vendas, correspondendo 207,9 milhões dos 343,3 milhões de unidades comercializados. Outros medicamentos para controle de colesterol e diabetes também estão entre as mais comercializadas. Os medicamentos genéricos estão disponíveis para diversos tipos de tratamentos de doenças, tais como: do sistema cardio circulatório, antiinfecciosos, aparelho digestivo/metabolismo, sistema nervoso central, antiinflamatórios hormonais e não hormonais, dermatológicos, doenças respiratórias, sistema urinário/sexual, oftalmológicos, helmínticoparasitários, oncológicos e contraceptivos entre outros. Com o vencimento das patentes, outros medicamentos genéricos destinados ao tratamento de doenças cardíacas e do sistema nervoso, brevemente estarão disponíveis.

O Diclofenaco de Potássio é um antiinflamatório não esteroidal, derivado do ácido fenilacético que apresenta atividade analgésica, antipirética e antiinflamatória, é um inibidor não seletivo da COX (ciclooxigenase) e possui fórmula química: $\mathrm{C}_{14} \mathrm{H}_{10} \mathrm{Cl}_{2} \mathrm{KNO}_{2}$ (1) adicionada aos excipientes ${ }^{1}$.

Os excipientes são agentes que têm a função de solubilizar, suspender, espessar, diluir, emulsificar, estabilizar, conservar, colorir, flavorizar e possibilitar a obtenção de formas farmacêuticas estáveis, eficazes e atraentes $^{2}$. Qualquer excipiente que não desempenhe bem algumas de suas funções, como dissolução e desagregação, poderá afetar diretamente a biodisponibilidade do fármaco. O Diclofenaco de Potássio é ainda um dos antiinflamatórios mais consumidos no Brasil.

A Microscopia Eletrônica de Varredura (MEV) é uma técnica de caracterização microestrutural capaz de produzir imagens de alta ampliação e resolução. Ela permite realizar uma análise multielementar com a adaptação de um Sistema de Espectroscopia de Energia Dispersiva (Energy Dispersive Spectroscopy -EDS) na câmara da amostra, com ele pode-se obter rapidamente informações sobre a morfologia e identificação de elementos químicos de uma amostra sólida. Suas aplicações são, comumente, nas áreas de: biologia, odontologia, farmácia, engenharia, química, metalurgia, física, medicina, geologia, etc ${ }^{3}$. É possível utilizar outras técnicas de análise para tal fim, a Fluorescência de Raios X (X Ray Fluorescence - XRF) é uma delas, contudo, devido limitações de detecção da maioria dos equipamentos de XRF, alguns elementos que fazem parte da composição dos medicamentos não são detectados, fazendo com que a XRF seja uma técnica complementar as outras ${ }^{4}$.

Tendo em vista, que a técnica de MEV/EDS permite uma análise multielementar rápida, a limitação de alguns equipamentos e o questionamento levantado por muitas pessoas, inclusive médicos, em relação à eficiência de um medicamento genérico em relação ao seu referência, realizou-se uma análise qualitativa dos medicamentos: genérico e similar do diclofenaco de potássio de $50 \mathrm{mg}$ e comparou-se com seu medicamento de referência.

Desta forma, pode-se observar se há diferenças significativas dos elementos ali encontrados, para 
posteriormente verificar a importância de algumas substâncias, ou até mesmo, alertar sobre a presença delas informando a população sobre a igualdade dos medicamentos genéricos, mediante essa técnica.

Com os resultados obtidos neste trabalho confirmou-se a eficiência da MEV/EDS para análise qualitativa de medicamentos tornando-a também uma técnica de análise complementar para tal fim.

\section{Microscopia Eletrônica de Varredura}

Os microscópios ópticos convencionais têm um aumento máximo de 2000 vezes, acima deste valor, detalhes menores são imperceptíveis ${ }^{3}$. A MEV pode produzir imagens com alta ampliação, 300 mil vezes. Isto porque a MEV utiliza feixes de elétrons no lugar de fótons, que são utilizados na microscopia óptica convencional. Na MEV, os elétrons são "gerados" a partir do aquecimento de um filamento de tungstênio (W) ou de hexaboreto de lantânio $\left(\mathrm{LaB}_{6}\right)$, por efeito termiônico. $\mathrm{O}$ feixe de elétrons é acelerado, aplicandose alta voltagem, semelhante ao que ocorre num tubo de raios $\mathrm{X}$ convencional. Este feixe passa por lentes condensadoras reduzindo o seu diâmetro. Em seguida, ele passa por uma lente objetiva que o focaliza sobre a amostra. Então, o feixe de elétrons interage com a região de incidência da amostra até uma profundidade que pode variar de $1 \mu \mathrm{m}$ a $6 \mu \mathrm{m}$ e depende da natureza da mesma. É na região de incidência, que irá gerar os sinais (elétrons e/ou ondas eletromagnéticas produzidas) que são detectados e utilizados para a formação da imagem e para a microanálise ${ }^{5}$. O feixe de elétrons ao colidir com os átomos da amostra pode arrancar elétrons da mesma, deixando o átomo energeticamente no estado excitado. Para retornar ao estado fundamental, o átomo emite energia na forma de raios $\mathrm{X}$ característico ${ }^{6}$. Estes raios $\mathrm{X}$ são detectados por um sistema de EDS instalado na câmara de vácuo do equipamento e sua energia é característica do átomo que a emitiu. Assim, é possível traçar um histograma da sua energia, em elétron volt (eV) em função do número de fótons recebidos por segundo (contagens).

\section{Inflamação}

A palavra inflamação ou flogose é proveniente do latim inflamare, que significa pegar fogo. É uma reação dos tecidos vascularizados a um agente agressor caracterizada pela saída de líquidos e de células do sangue para o interstício, desencadeada pela liberação de mediadores químicos originados nas células migratórias e nos tecidos lesados ${ }^{7}$. Diferentes agressões biológicas, físicas ou químicas constituem o que denominamos como agentes inflamatórios ou flogógenos. Estes agem nos tecidos e promovem uma resposta imediata de alarme (liberação de moléculas sinalizadoras de agressão), as quais induzem a liberação de outras moléculas geneticamente conhecidas como mediadores da inflamação, que resultam em modificações na microcirculação necessárias para a saída de plasma e de leucócitos dos vasos e em estímulos para reparar os danos produzidos pelas agressões.

A resposta inflamatória está associada aos processos de reparo no organismo e representa um processo ao mesmo tempo defensivo e reparador ${ }^{7}$. Numerosos estímulos podem desencadear os eventos que compõem o processo inflamatório como, por exemplo, agentes infecciosos, lesão térmica ou lesão por agentes físicos e cada um desses estímulos irá provocar um padrão característico de resposta.

Em nível macroscópico sinais clínicos distintos acompanham esta resposta, são eles: eritema vermelhidão provocada em decorrência da dilatação vascular; edema - devido ao acúmulo extravascular de fluído; calor - devido ao aumento do fluxo sanguíneo no local lesionado; dor - provocada pelo acúmulo de fluido intersticial e devido a mediadores químicos como a bradicinina; e perda da função.

\section{Anti-inflamatórios}

Os anti-inflamatórios podem ser classificados de acordo com duas categorias: os esteróides ou corticosteróides (AIES) e os não-ésteróides (AINE). Os AINE atuam como analgésicos, antitérmicos e antiinflamatórios através de um mecanismo de ação geral que se dá por inibição periférica e central da atividade das enzimas ciclooxigenase-1 (COX-1), expressa constitutivamente na membrana das células e responsável pela síntese de prostaglandinas envolvidas em funções homeostáticas (agregação plaquetária, manutenção da filtração glomerular e efeito citoprotetor da mucosa gástrica), e ciclooxigenase-2 (COX-2), induzida por determinados fatores séricos, com subsequente diminuição da biossíntese e liberação dos principais mediadores da inflamação, dor e febre, conhecidos como eicosanoides ${ }^{8}$.

O mecanismo de ação dos AINE consiste da inibição das enzimas COX1 e COX2 e, consequentemente, na diminuição da produção de prostaglandinas e redução da inflamação, dor e febre. Atualmente, os AINE disponíveis promovem inibição tanto da cicooxigenase-1 (COX-1; constitutiva) como a da ciclooxigenase-2 (COX-2; induzida na presença de inflamação) inibindo, portanto, a síntese de prostaglandinas e tromboxanos ${ }^{8}$. 


\section{MATERIAL E MÉTODOS}

As análises foram realizadas no Laboratório do Instituto Federal de Educação, Ciência e Tecnologia do Rio de Janeiro, Campus Paracambi - RJ.

\section{Amostras}

O medicamento utilizado para análise foi o Diclofenaco de Potássio $50 \mathrm{mg}$, um derivado do ácido fenilacético que apresenta atividade analgésica, antipirética e anti-inflamatória, não seletivo como inibidor da $\mathrm{COX}$ e pertencente à classe dos antiinflamatórios não esteroides ${ }^{1}$. Os genéricos e similares do fármaco em questão, segundo a ANVISA, devem obrigatoriamente apresentar o mesmo princípio ativo, na mesma concentração do medicamento referência. No entanto, os excipientes não precisam ser idênticos em ambos os produtos 2 .

O medicamento em estudo apresenta em sua composição $50 \mathrm{mg}$ de Diclofenaco de Potássio adicionados aos excipientes. Foram utilizados medicamentos de três empresas farmacêuticas distintas, o similar e o referência, todos com o mesmo vencimento.

\section{Irradiação}

As amostras foram irradiadas com um microscópio eletrônico de varredura, sistema comercial portátil de bancada TM-3000 Hitachi High Technologies. Ele tem como características tensão de aceleração variável 5 a $15 \mathrm{kV}$, magnificação de até 30.000 vezes, sistema de EDS sem necessidade de refrigeração com nitrogênio líquido, análise elementar por raios $\mathrm{X}$, além de fácil operação.

Para irradiar as amostras, primeiramente abriuse a câmara de vácuo, para isto pressionou-se o botão EVAC/AIR. Em seguida colocou-se o medicamento no porta-amostra, fechou-se a câmara de vácuo pressionando-se novamente o botão EVAC/AIR para evacuar a câmara. Deste modo os elétrons incidentes e os raios X característicos emitidos não perderiam suas energias iniciais

Para iniciar-se a irradiação utilizaram-se os softwares que permitiram dosar a técnica apropriada e análise dos dados. Este último é feito por meio do software Quantax 70. Primeiro irradiou-se os medicamentos diretamente, em seguida, raspou-se os mesmos medicamentos retirando-se o revestimento para que suas espessuras não interferissem nas medidas. Foram realizadas três medidas para cada amostra, em pontos distintos, durante $300 \mathrm{~s}$, com energia de $15,0 \mathrm{kV}$, magnificação de 1000 vezes.

\section{RESULTADOS E DISCUSSÃO}

Nas figuras 1, 2, 3, 4 e 5 é possível observar claramente a distinção dos elementos presentes no revestimento do medicamento e dos elementos que pertencem ao medicamento sem o revestimento.

$\mathrm{O}$ medicamento referência não apresentou cálcio $(\mathrm{Ca})$ quando analisado com o revestimento, isso se deve ao fato da camada protetora obtida por drageamento ser espessa ao ponto dos fótons dos raios $\mathrm{X}$ característicos do Ca não a atravessarem.

Ao retirar o revestimento todos os genéricos apresentaram-se bem semelhantes qualitativamente quando comparados ao medicamento de referência, conforme mostrado nas Figuras 1, 2, 3, 4 e 5.

A Lei 9787/99 da ANVISA/MS diz que os excipientes não precisam ser idênticos em ambos os produtos. Entretanto, os excipientes presentes em uma formulação farmacêutica podem afetar a dissolução do fármaco, e consequentemente a velocidade e quantidade pelas quais o mesmo estará disponível para ser absorvido $^{10}$. Em alguns casos, a escolha do excipiente pode ser interessante para alterar o tempo que um fármaco permanece no organismo.

Os elementos encontrados no medicamento referência correspondem aos seguintes excipientes contidos na bula: $\mathrm{Na}$ (carboximetilamido sódico), $\mathrm{Mg}$ (estearato de magnésio), $\mathrm{Si}$ (Dióxido de silício), $\mathrm{Cl}$ e K (princípio ativo), Ti (dióxido de titânio) e Fe (óxido de ferro vermelho). Para o Genérico "A", os elementos encontrados, segundo a bula, são: $\mathrm{Na}$ (amidoglicolato de sódio), Mg (estearato de magnésio), Al (corante alumínio laca amarelo crepúsculo 6), $\mathrm{P}$ (fostato de cálcio dibásico), $\mathrm{Cl}$ e K (princípio ativo), Ti (dióxido de Titânio), Fe (óxido de ferro vermelho). Para o Genérico "B", os elementos encontrados, segundo a bula são: $\mathrm{Na}$ (croscarmelose sódica), Mg (estearato de magnésio), Si (dióxido de silício), $\mathrm{P}$ (fosfato de cálcio dibásico dihidratado), $\mathrm{Cl}$ e $\mathrm{K}$ (princípio ativo), Ca (fosfato de cálcio dibásico di-hidratado), Ti (dióxido de titânio) e Fe (óxido de ferro vermelho). Já no Genérico "C", os elementos encontrados são: Mg (estearato de magnésio), Si (dióxido de silício), Al (copolímero de dimetilamino etilmetacrilato), Ti (dióxido de titânio), Fe (óxido férrico vermelho), $\mathrm{Na}$ (croscarmelose sódica), $\mathrm{P}$ (fostato de cálcio dibásico), $\mathrm{Cl}$ e $\mathrm{K}$ (princípio ativo) e Ca (fosfato de cálcio dibásico).

$\mathrm{O}$ excesso de cálcio no medicamento como excipiente pode diminuir a absorção do fármaco, já que o excipiente em questão é hidrofóbico. Aumentando assim, o início do tempo de ação. Em alguns medicamentos isso pode ser interessante para diminuir os riscos de intoxicação medicamentosa. 


\section{Medicamento Referência}

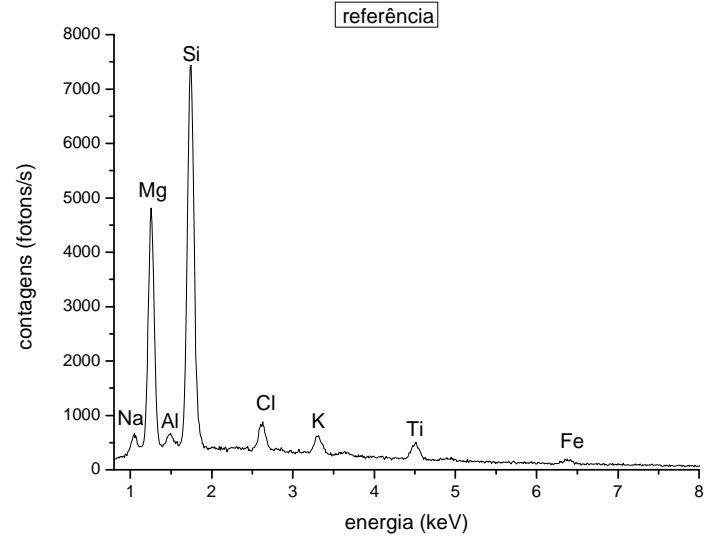

(a)

\section{Medicamentos Genéricos}

\section{Genérico A}

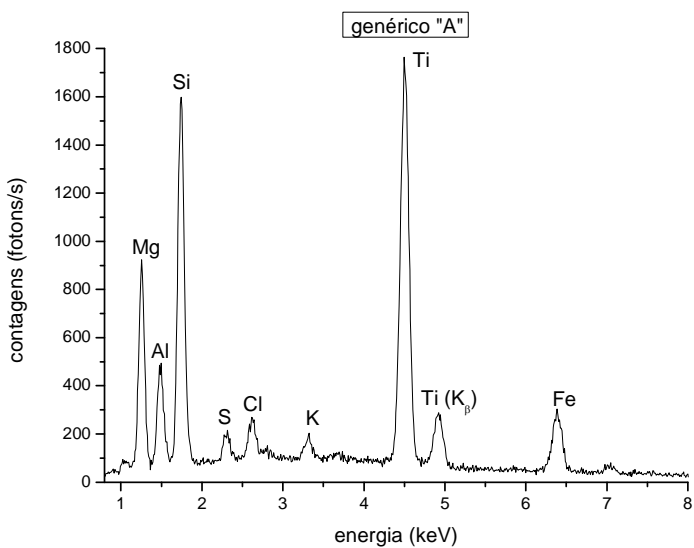

(a)

Figura 2. Espectros referente ao medicamento genérico do Diclofenaco de Potássio 50 mg da empresa A: (a) com revestimento (b) sem o revestimento.

(b)

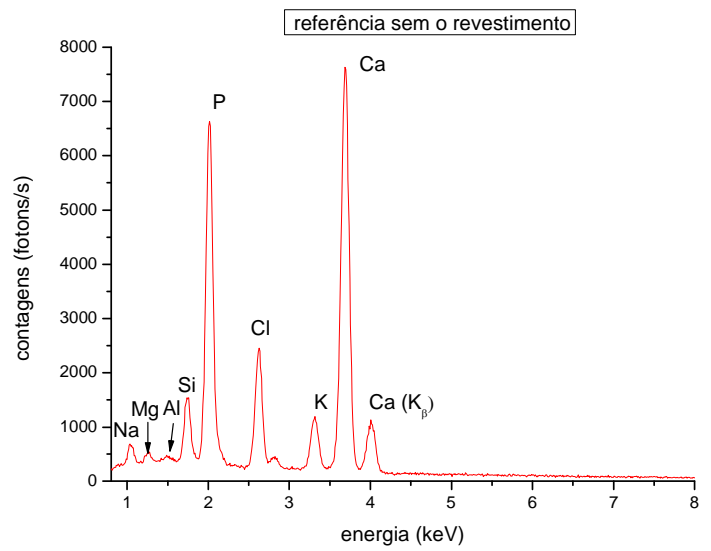

(b)

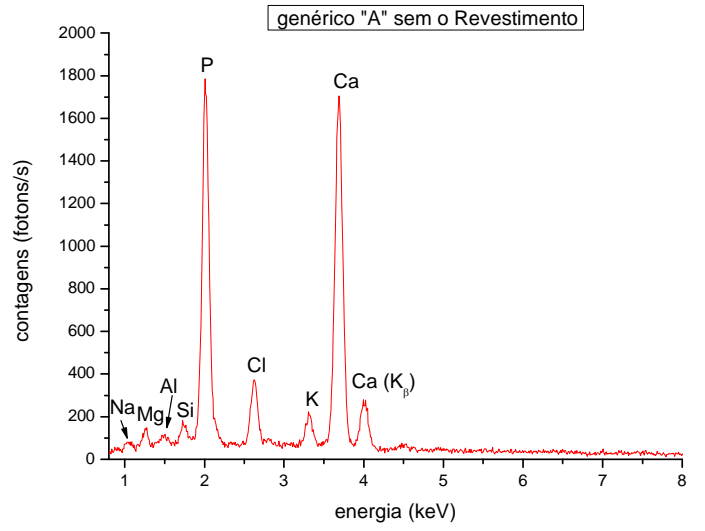




\section{Genérico B}

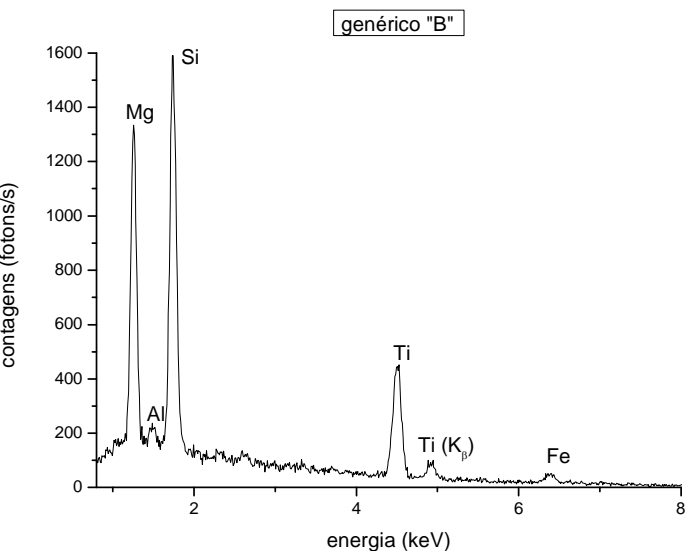

(a)

Figura 3. Espectros referente ao medicamento genérico do Diclofenaco Potássico 50 mg da empresa B: (a) com o revestimento e (b) sem o revestimento.

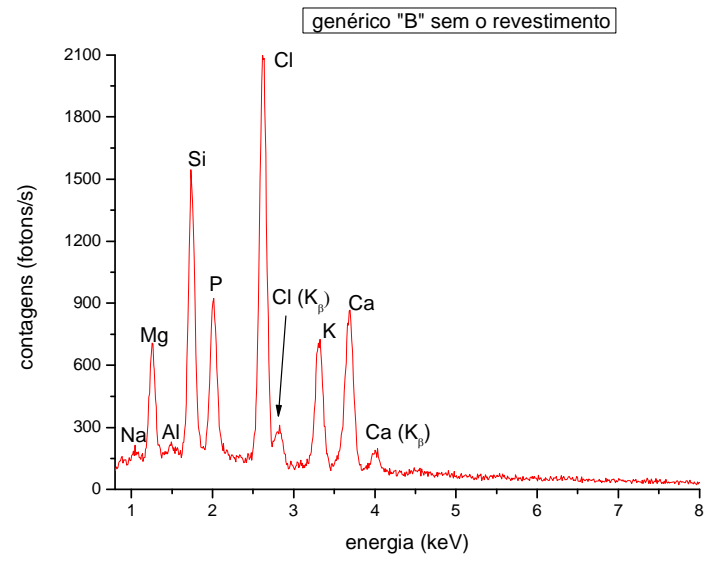

(b)

\section{Genérico C}

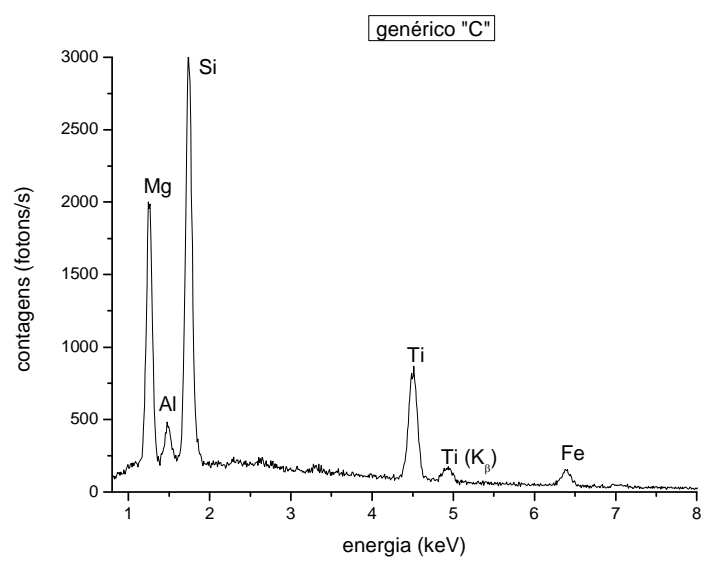

(a)

Figura 4. Espectros referente ao medicamento genérico do Diclofenaco de Potássio 50 mg da empresa C: (a) com o revestimento e (b) sem o revestimento.

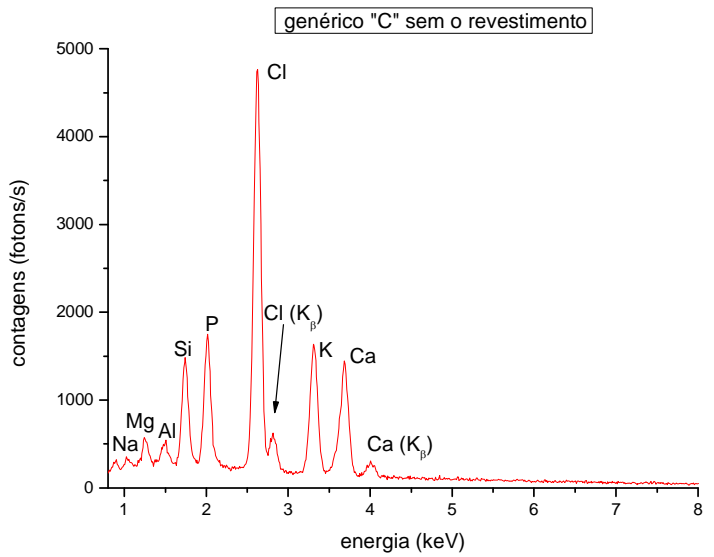

(b) 


\section{Medicamento Similar}

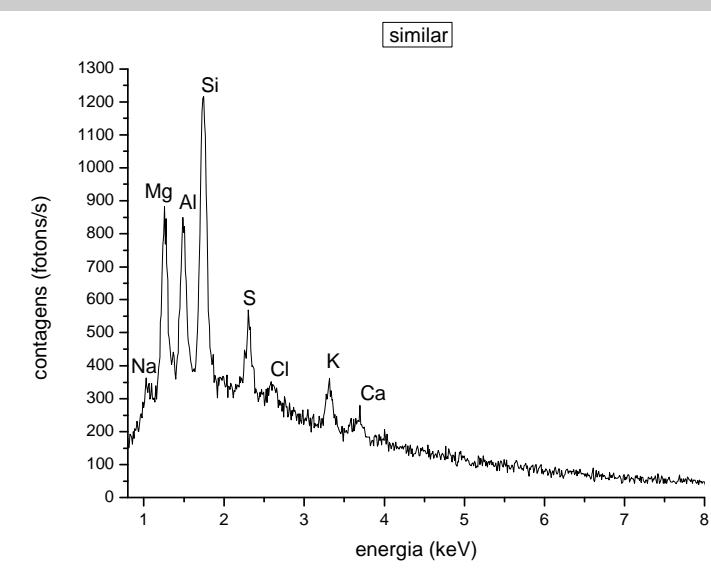

(a)

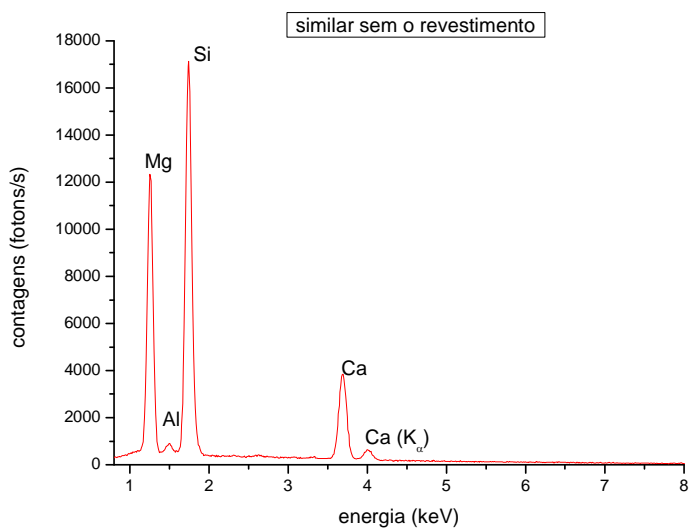

(b)

Figura 5. Espectros referente ao medicamento similar do Diclofenaco de Potássio 50 mg: (a) com o revestimento e (b) sem o revestimento.

\section{CONCLUSÃO}

Em todos os fármacos analisados o equipamento detectou os excipientes, mostrando ser uma técnica muito eficiente quando comparada com a análise por XRF já realizada para o mesmo fármaco. A MEV pode ser utilizada como uma técnica complementar para detecção de elementos presentes em medicamentos para o controle da composição de fármacos.

Contudo, sabe-se que para verificar a reação ou tempo de resposta do medicamento no organismo

\section{REFERÊNCIAS}

1. Teixeira MJ, Teixeira WGJ, Andrade DCA. Tratamento farmacológico da dor musculoesquelética. Rev. Med. 2001; 80(ed.esp.pt.1): 179-244.

2. Ministério da Saúde (Br), Agência Nacional de Vigilância Sanitária, Lei no 9.787, de 10 de fevereiro de 1999.

3. Dedavid BA, Gomes CI, Machado G. Microscopia Eletrônica de Varredura: Aplicações e preparação de amostras: materiais poliméricos, metálicos e semicondutores. Porto Alegre: EDUPUCRS; 2007. 60p. do paciente é necessária uma pesquisa adjacente, com amostras sanguíneas dos pacientes, massa, sexo, para comprovar as diferenças na biodisponibilidade dos medicamentos. Entretanto, este não foi o objetivo do trabalho.

É importante informar que o presente trabalho, não teve como objetivo, denegrir nenhuma indústria farmacêutica, nem tanto, mostrar a superioridade de uma em relação à outra, mas sim mostrar que a presente técnica pode ser utilizada pelos laboratórios para fins de análise multielementar.
4. Luiz LC, Batista RT, Oliveira LF, Santos RS, Nascimento ECS, Brandão DL, Freitas CBL, Anjos MJ. Utilização de um Sistema Portátil de Fluorescência de Raios X para Análise Multielementar de Medicamentos: Genéricos e Referência. Physicae. 2014; 10(10): 26-32.

5. Silva D. Microscopia Eletrônica de Varredura, Notas de Aula. Unicamp. Disponível em: http:// www.ige.unicamp.br/site/aulas/186/MevAula1.pdf. Acesso em: 11 de março de 2015. 
6. Luiz LC. Introdução à Física Radiológica em Radiologia Médica e Equipamentos Radiológicos. Rio de Janeiro: Edição Independente; 2011.

7. Bogliolo L, Brasileiro Filho G. Patologia. 6ª ed. Rio de Janeiro. Guanabara Koogan; 2000.

8. Goodman LS, Gilman AG. As bases farmacológicas da terapêutica. $10^{\mathrm{a}}$ ed. Rio de Janeiro: McGraw-Hill Interamericana do Brasil Ltda; 2005.

9. Ferreira AO. Guia prático da farmácia magistral. $3^{\mathrm{a}}$ ed. São Paulo: Pharmabooks; 2008.

10. Storpirtis S, Marcolongo R, Gasparotto FS, Vilanova CM. A equivalência farmacêutica no contexto da intercambialidade entre medicamentos genéricos e de referencia: Bases técnicas e científicas. Infarma. 2004;16(9-10): 51-56.

11. Oliveira, JC. Monitoramento e vigilância tecnológica do mercado de medicamentos genéricos no Brasil no período de 2000 a 2015. [monografia]. Brasília: UnB; 2015. 47p. Disponível em: http://bdm.unb.br/bitstream/10483/10850/ 1/2015 JoyceCarladeOliveira.pdf Acesso em: 30 de dezembro de 2015 .
12. Revista do Farmacêutico. 15 anos de medicamentos genéricos. №117. jul-ago/ 2014. 22-23. Disponível em: http://portal.crfsp.org.br/images/stories/revista/rf117/ rf117.pdf Acesso em: 30 de dezembro de 2015.

\section{Correspondência}

Leandro da Conceição Luiz

Faculdade Bezerra de Araújo. Rua Viúva Dantas, 417 Campo - Rio de Janeiro - Rio de Janeiro - Brasil

CEP 23052-090

E-mail:mrleandroluiz@hotmail.com 